\title{
Diálogo para la reflexión: compartiendo la experiencia de aula desde el proyecto pedagógico
}

María Margarita Urdaneta Benavides

Universidad Nacional, División de Educación Básica, Heredia; margarita_urdaneta@hotmail.com

Recibido: 27 de mayo del 2014

Corregido: 27 de noviembre del 2014

Aceptado: 05 de diciembre del 2014

"Detrás de lo ordinario se halla lo extraordinario."

W. Jackson.

\section{Resumen}

El presente ensayo nace de la vivencia como profesora tutora del curso de Proyecto Pedagógico, curso que coordina la experiencia de intervención pedagógica que es parte de la formación de los estudiantes del nivel de Bachillerato de la carrera de Pedagogía con énfasis en Educación Preescolar, de la División de Educación Básica de la Universidad Nacional.

El interés sobre la posibilidad de lograr mediar procesos; que permitan, a los estudiantes que vivencian la intervención pedagógica, acercarse al reconocimiento de sus estrategias reflexivas y meta-cognitivas (a partir de la socialización de los momentos de interacción en el aula preescolar en los que el profesor tutor acompaña al estudiante), ha motivado a retomar la experiencia, a través de la reflexión personal que se presenta en este ensayo.

De manera que, a partir de una visualización de las vivencias desde la labor docente en el aula universitaria, se pretende ilustrar las estrategias para la reconstrucción y reflexión posterior a la acción, el futuro docente sea quien logre visualizarse a partir de su propia mirada, $y$, no únicamente, a través de la mirada del profesor tutor.

Palabras clave: mediación docente, meta-cognición, reflexión, socialización del aprendizaje, intervención pedagógica.

\begin{abstract}
Dialogue for reflection: sharing the experience of classroom from the pedagogical project

The present essay was born of experience as professor tutor of the course of Pedagogical Project, that course coordinated the pedagogical intervention as an experience that is part of the training of the students of the Bachelor level of the career of teaching with emphasis on preschool education of the Division of Basic Education from the National University of Costa Rica.

The interest about the possibility of mediating processes, that allow students to experience the pedagogical intervention approach to the recognition of their reflective strategies and metacognitive, starting the socialisation of the moments of interaction in the preschool classroom where the professor tutor accompanies the student, has motivated to return to the experience. So, from a display of experiences from the teaching in the University classroom, is intended to reach illustrate strategies for reconstruction and later reflection to action, the future teacher wherever that achieves displayed from your own eyes, and not only through the eyes of your teacher.
\end{abstract}

Key words: Teaching mediation, metacognition, reflection, socialization of learning, pedagogical intervention. 


\section{INTRODUCCIÓN}

El Centro de Investigación y Docencia en Educación (CIDE), de la Universidad Nacional (UNA), es la División de Educación Básica (DEB) la unidad académica encargada de coordinar todo lo referente a la formación de profesionales en Pedagogía con énfasis en Educación Preescolar, Pedagogía con énfasis en I y II Ciclo, Educación Especial y Enseñanza del Inglés, y es, por consiguiente, desde donde se plantea la naturaleza de la intervención pedagógica de cada plan de estudios.

La inserción de estudiantes, desde los inicios de su formación, en la realidad de los espacios destinados a la atención de niños y niñas, se visualiza claramente, desde las Áreas y Principios de la carrera de Pedagogía con Énfasis en Educación Preescolar, Universidad Nacional (UNA). (2012), como un eje medular a lo largo de todas las experiencias del aula universitaria. Dicho eje, tal y como se pretende desde el plan de estudios antes mencionado, "busca que el estudiante parta de sus experiencias prácticas, como un medio para investigar, analizar, y generar procesos tendientes [sic] a lograr cambios en la realidad educativa”. (Universidad Nacional (UNA), 2012, párr. 9). Con igual nivel de importancia el eje meta-cognitivo, presente en las Áreas y principios de la carrera de Pedagogía con énfasis en Educación Preescolar como, "la conciencia y consideración por parte de una persona de sus propias estrategias y procesos cognitivos" (Universidad Nacional (UNA), 2012, párr. 9). También señala, "se busca además, que la meta-cognición se plasme como una estrategia de formación docente, y que sea parte de los procesos de enseñanza aprendizaje. (Universidad Nacional (UNA), 2012, párr. 9). El tema de la formación del docente reflexivo ha apasionado a muchos autores a través del tiempo, siempre en la búsqueda de trascender de una racionalidad técnica a un planteamiento donde sea la reflexión quien lleve al profesional a un diálogo entre teoría-práctica, que provenga de un replanteamiento de las acciones que -como profesional- practique en el aula, y no desde una concepción de laboratorio del campo de acción profesional, donde la teoría se lleva para ser probada, en ausencia de cualquier tipo de relación con el contexto.

Como lo señala Sanjurjo (2002), el proceso de formación docente, y específicamente la intervención pedagógica, representa una oportunidad de vivenciar procesos reflexivos, como lo menciona la autora,

\footnotetext{
“a. Es una situación novedosa y compleja. b. Se trata de una experiencia supervisada por los docentes que acompañan a los residentes y que tienen $[s i c]$ como función confrontar pensamiento y acción. c. Los residentes registran sus acciones pedagógicas, deben justificarlas y reflexionar sobre ellas". (p. 11)
}

Es entonces el momento propicio para que, no solo el estudiante reflexione de un proceso que el mismo está propiciando desde su práctica, sino que es una oportunidad para que el profesor tutor encuentre una posibilidad de mediar en esa construcción de procesos reflexivos y meta-cognitivo.

La formación de formadores es una tarea que requiere despertar en los involucrados tanto estudiantes, como académicos, la necesidad de propiciar experiencias que conduzcan llevar a una comprensión de todas las situaciones que se presentan en el aula y valorarlas como una oportunidad de aprendizaje, a partir de la reflexión de la acción de manera consciente, evidenciándose esa reflexión en la incorporación de cambios en las prácticas.

Es justamente el despertar a la reflexión, en aras de procurar trascender de lo aparentemente ordinario de la experiencia vivida en el aula universitaria con los estudiantes del curso Proyecto Pedagógico, lo que se pretende compartir en el desarrollo de este ensayo.

Ahora bien, en esa reflexión surgen algunas preguntas ¿cómo se puede lograr una mediación que genere un aporte para fortalecer la propia consideración de los procesos meta-cognitivo de los estudiantes a partir de la visualización y reflexión de su proceso de práctica?, ¿Qué estrategias serían pertinentes para acercar a los estudiantes a esa reflexión? Estas preguntas fueron formuladas para iniciar un diálogo propio evocando lo vivido. 


\section{- Desde la experiencia y la reflexión de profesora tutora}

Desde el marco que representa el Plan de Estudios de la carrera de Educación Preescolar en la labor académica, nace la experiencia de realizar una mediación en los procesos de reflexión y metacognición construidos por las estudiantes del curso Proyecto Pedagógico del año 2012.

La Universidad Nacional (UNA). (2012), desde los descriptores de los cursos de la carrera de Pedagogía con énfasis en Educación Preescolar el curso de Proyecto Pedagógico especifica que,

(...) promueve en el estudiante la sistematización de las experiencias prácticas en el aula preescolar como instrumento para la investigación, análisis y construcción de procesos tendientes a lograr cambios en la realidad educativa. Se requiere en práctica la implementación de un proyecto concreto de carácter pedagógico, para lo cual es necesaria la inserción del estudiante en una institución de preescolar, de manera sistemática. (Apartado de descripción del curso, párr. 1).

A partir de la descripción anterior, se entiende que los estudiantes inician sus prácticas en diferentes instituciones de atención de niños y niñas en edad preescolar, lo que les permite interactuar con realidades distintas, en busca de un diagnóstico inicial que los llevará al planteamiento de un proyecto, pertinente a las personas y al contexto que han conocido. Por la naturaleza desde la que está concebida la práctica del nivel de bachillerato, los estudiantes tienen la oportunidad, no solo de intercambiar experiencias con niños y niñas, sino también con personas que participan en el proceso de gestión del proyecto pedagógico que ellos lideran.

Es relevante visualizar además que "Este curso coordina las experiencias de intervención pedagógica. Así mismo, es el curso que se considera dentro de la estructura del plan de estudios como la práctica profesional supervisada (PPS)" (Universidad Nacional (UNA), 2012, apartado de Descripción del curso, párr. 2).
Desde esta concepción de la práctica profesional supervisada en el nivel, la labor del profesor tutor pretende recabar información desde la visión de los diferentes participantes de Proyecto Pedagógico, sobre ¿cómo los estudiantes logran ejercer sus funciones tanto administrativas, organizativas y motivadoras como pedagógicas, del proyecto que ellos mismos han diseñado y se encuentra ejecutado?

Pues bien, la experiencia de la búsqueda de esta respuesta es riquísima y plantea la necesidad de ser diferente a otros procesos, pues se debe concebir como una mediación que permita, no solo obtener información para la valoración del quehacer de los estudiantes en el espacio y situaciones en los que ellos son protagonistas, sino propiciar, mediante diferentes recursos, el auto-reconocimiento del proceso meta-cognitivo y la reflexión en los estudiantes. Se puede mencionar que el diálogo mediante el cual socializa la experiencia, así como la reflexión de la acción mediante el recuento escrito de lo vivido por medio de la crónica, son los medios que se han utilizado como un acercamiento a la construcción propia que hace el estudiante de esta etapa de la formación profesional tan relevante y significativa para su aprendizaje como lo representa la práctica profesional supervisada.

\section{- Conversando sobre lo vivido}

Con este fin se plantea el espacio de diálogo con cada uno de los estudiantes, momento después de que se ha realizado la visita de observación respectiva. Este espacio puede ser definido como un momento de socialización de la experiencia vivida por el estudiante y el profesor tutor, socialización que, como lo menciona Sanjurjo et ál., (2009) facilita el aprendizaje por medio del intercambio de experiencias y situaciones, a través del diálogo conjunto.

En el diálogo sobre la experiencia vivida los estudiantes expresan la necesidad de contar con otra visión de la situación en la que han sido partícipes, para retomar aspectos olvidados, para compartir emociones, creencias y conocimientos construidos, como algunos señalaron. Sierra et ál., (2002) menciona; que el lenguaje es un instrumento de 
comunicación y, además, es vital en todo proceso de construcción de conocimientos y pensamientos ( $\mathrm{p}$. 194). El lenguaje es, entonces, un medio fundamental para que el tutor comprenda, analice y guíe a los estudiantes en su afán por perfeccionar los procesos de aprendizaje de los que son partícipes. Agrega, además; "con él representamos e interpretamos la realidad social, natural, política..." (Sierra et ál., 2002, p. 194-195).

Por lo tanto, el profesor tutor y el estudiante tienen diferentes perspectivas de una situación que ha sido observada y vivenciada por ambos, y que durante la socialización, mediante el diálogo de la experiencia, pretenden generar un compartir para propiciar una situación que busca despertar, como lo señala Sanjurjo (2002), la capacidad de evaluar, planificar y regular el proceso de aprendizaje propio, mediante la autovaloración que logre el estudiante.

La conversación sobre esa situación inicia con una evidente ansiedad de aprobación por parte del estudiante, luego de vivir la intervención en el aula y; el nerviosismo. La idea de solo ponderar el aspecto evaluativo persiste, a pesar de que existe sensibilización previa de parte del equipo de profesores del curso de Proyecto Pedagógico, de que el momento de la intervención debe significar, para ellos, una oportunidad para reflexionar y aprender.

Las expresiones que dibujan este primer momento de tensión y ansiedad se muestran con claridad, por medio de frases que sugieren la idea de que ellos esperan un veredicto final o una disculpa por situaciones críticas presentes en la experiencia, "profesor, cómo estuvo...", "los chicos nunca se portan de esa forma", "que cree que puedo hacer con..." La respuesta a estas frases no se manifiesta como ellos lo esperan, todo lo contrario, inician con,

¿Podríamos conversar sobre lo que acaba de suceder?, hagamos una reconstrucción, ¿usted comienza?, la propuesta surge con el objetivo de propiciar una oportunidad de que el estudiante se visualice desde afuera de lo que acaba de vivir en su práctica, y lograr ubicarla en un lugar desde el que pueda ser consciente de sus propias acciones e intente verbalizar, como explica Schön (citado por Sanjurjo et ál., 2009), el conocimiento en acción, que se constituye en la base de todas las acciones y su descripción es parte de la construcción de los conocimientos que se profundizan con la reflexión.

\section{- Reconociéndose en la acción}

Los estudiantes inician con la reconstrucción detallada de lo sucedido, se aprecian señales de una valoración propia y una identificación de los involucrados y su roles en la acción. Al respecto, Sierra et ál., (2002) plantea “... la apropiación del lenguaje, por los estudiantes, es una acción pedagógica que va dirigida al desarrollo del pensamiento autónomo y crítico como valor formativo..."(p. 195), por lo que no es entonces la visión del profesor tutor la que se trata de imponer ante la del estudiante, sino que es desde la apropiación de la experiencia, que el estudiante comenzará a reflexionar, fomentando la autocrítica que lo llevará a las causas para que en uso de su autonomía replantee su acción.

Posteriormente, se ahonda en llevar al estudiante a que se observe el mismo, que valore su rol como docente en las acciones y responsabilidad que tiene dentro de la dinámica en la que ha participado. Esta mediación se presenta como una oportunidad de guiar a la conciencia, a la reflexión, al análisis y para reconocer, como tutor, la estrategia de acción que el estudiante practica para hacer una guía pertinente del proceso. "...conocer los análisis, reflexiones, valoraciones, aportaciones, observaciones y puntos de vista de los estudiantes constituye, en primer lugar, una entrada de información que hace aumentar el conocimiento disponible para cambiar las condiciones didácticas, metodológicas e interpersonales..." (Sierra et ál., 2002, p.201).

El conocer la estrategia del estudiante lleva a considerar diferentes caminos para orientar las futuras acciones del estudiante en el aula. Un diálogo sostenido con un estudiante ilustra la dinámica de ese compartir: "La idea era hablar con los chicos de la tarea, como les había ido con los papás y familiares, luego retomar cómo se ahorraba el agua y por qué era importante cuidarla, ipero colapsé!... Nuevamente se presenta la oportunidad de llevarlo a reconocer qué hizo en el momento, ¿cuándo se da cuenta de que 
"colapsa" qué piensa, y qué hace finalmente?: "trato de mantener la calma, concentrarme y acordarme de cómo estaba planeado" El recapitular las decisiones que se toman en la acción permiten reconocer cuál es la manera en que se toman decisiones en el momento, y cuestionarse estas decisiones o reflexionar cómo se llegó a ellas. “... el estudio de la toma de decisiones por parte del docente es de crucial importancia para comprender el pensamiento del profesor..." (Sanjurjo, 2002, p. 45). Es así como, poco a poco, comienzan a emerger, desde la visión y reflexión de los estudiantes, sus propias propuestas de cambio para las próximas intervenciones.

El recuento escrito de lo vivido, expresado por medio de una crónica, desde una voz más personal e íntima de los procesos que los estudiantes van construyendo, permite profundizar en el acompañamiento de la vivencia.

\section{- Manifestando las estrategias para la reflexión}

Una manifestación escrita según Sanjurjo et ál., (2009), tiene un impacto en la enseñanza y el aprendizaje porque los docentes recuperan su protagonismo en su práctica, al expresarse como autores del relato de una realidad propia. Los estudiantes reconocen que al expresar de manera escrita su realidad, en una estrategia como el -diario del profesor- les permite entrar en un proceso de autoevaluación, "De igual manera el uso de un diario del profesor es de gran ayuda para este aspecto debido a que de esa manera se puede observar en qué se va mejorando y qué nos falta por mejorar". A lo largo del proceso van surgiendo diálogos internos, generados en un proceso reflexivo que se ve plasmado en lo escrito, "He ido aprendiendo a relajarme por unos instantes, mirar adentro de mí, analizar qué estoy sintiendo y pensado para buscar la mejor solución", comparte un estudiante en un momento del proceso de su práctica.

Como lo comparte Sanjurjo et ál., (2009), la meta-cognición concibe el evaluar, planificar y regular el propio proceso de aprendizaje, mediante la autovaloración de los procesos que se vivencian de manera personal, y estos procesos deben lograr ser expresados por las personas que los viven.

En el marco de la conversación con el estudiante, se busca llevarlo a una revisión de los hechos en los que, primero se logre visualizar como parte de esa vivencia, permitiendo reconocerse en los momentos claves de sus acciones, para luego traducirlos en posibles acciones propuestas por el mismo y no por un observador externo, quien lo que procura en este caso es comprender y guiar al estudiante en ese encuentro con los replanteamientos de su práctica, durante y posterior a la acción, así como, también, llevarlo a dialogar con el mismo, para que reconozca y valide sus propias estrategias para la reflexión y meta-cognición.

\section{CONCLUSIONES}

\section{- Después de la experiencia, las reflexiones propias}

De la vivencia de diálogo, entre el estudiante y el profesor tutor en busca de la reflexión que lleva a generar cambios evidentes en la práctica, es inevitable el auto-cuestionamiento sobre los retos que se presentan, relacionados con la intervención pedagógica como eje fundamental en la formación de los estudiantes. Las preguntas surgen a modo de retos, pues representan la reflexión personal sobre posibles vacíos en el manejo de la intervención pedagógica como una herramienta valiosa en la formación docente. Además, las preguntas guían el diálogo personal que surge casi de inmediato a la conversación con el estudiante.

Los cuestionamientos son directos y relacionados con la oportunidad de construir aprendizajes a partir de las vivencias de la intervención pedagógica y lo que podría representar para la formación de los futuros docentes. Considerando la riqueza de la conversación con el estudiante y la lectura de sus crónicas personales surge la primera interrogante.

¿Cómo lograr que la experiencia de intervención pedagógica sea visualizada como una herramienta para el aprendizaje a lo largo de la formación, y no 
solo se haga presente como una tarea central de un curso específico?

Jackson (1998) señala que "casi tan importante como la propia observación es la exigencia de mantener una mente abierta ante lo que vemos..." (p. 207), casi de inmediato, el papel de los docentes que acompañan el proceso toma relevancia, como formadores ¿le estaremos otorgando a la intervención pedagógica, a lo largo del proceso de formación, la importancia que esta merece, no solo desde la vivencia de conocer otras realidades, sino en términos de propiciar habilidades en los estudiantes, que los lleve a sentir el ambiente del aula, reconocerse dentro de la dinámica, observar más allá de lo que creemos rutinario, a partir de la cercanía con esas realidades?

Dicho autor apunta sobre este aspecto que se presenta como un reto dentro del diálogo interno, "muchos aspectos de nuestra vida en el aula parecen triviales. Y en cierto sentido lo son. El entendimiento pleno de su importancia empieza a surgir cuando reflexionamos sobre su presencia acumulativa" (p. 207). Se reafirma la búsqueda de generar habilidades en los estudiantes que les permitan miradas más profundas en cada una de las oportunidades de intervenir en el aula desde la experiencia de formación universitaria.

¿Cómo favorecer, como profesores formadores, una mediación cercana, pertinente y sensible a los procesos que viven los estudiantes en los espacios de intervención pedagógica, para estimular en ellos procesos de auto-diálogo y reflexión, que los lleve a niveles de lectura de su práctica mucho más profundas y menos descriptivas?

La importancia de la reflexión y el reto de acercarse a los estudiantes para buscar una mediación pertinente a las necesidades y vivencias para ayudar a mostrar las formas propias de reflexión es abordado por los autores Gimeno y Pérez (2000) "la reflexión implica la inmersión consciente del hombre en el mundo de su experiencia, un mundo cargado de connotaciones, valores, intercambios simbólicos, correspondencias afectivas...” (p. 417). Se está ente el reto de lograr que los estudiantes sean conscientes de esa realidad, desde los primeros momentos y de manera progresiva en su formación con la guía cercana de los profesores formadores.

“...el inicio temprano de la formación docente en nuestras primeras experiencias como alumnos. En ellas vamos internalizando acríticamente, teorías acerca del enseñar y del aprender que se arraigan muy firmemente se transforman en supuestos básicos, subyacentes y muy difíciles de modificar..." (Sanjurjo, 2002, p. 38)

La práctica profesional supervisada, es un momento en el cual los profesores pueden reorientar muchas prácticas que los estudiantes han incorporado a su sistema de acción y que pareciera en ocasiones que se han alimentado más por supuestos personales que por conocimientos que provengan de la relación teoría-práctica que se ha construido a partir de toda la formación, desde los niveles iniciales.

La riqueza de la socialización de las experiencias de los estudiantes desde sus vivencias durante la intervención pedagógica en el curso de Proyecto Pedagógico plantea un nuevo reto y deja ver el interés en cómo lograr implementar la socialización de las experiencias de intervención pedagógica vividas por los estudiantes a lo largo de la formación, como una acción constante que permita el análisis de la experiencia de intervención, conjugando los aportes teóricos de la formación profesional y las acciones vivenciadas por los estudiantes; para dar origen a discusiones más profundas construidas en comunidad, guiadas no solo por los cuestionamientos de los profesores, sino por los cuestionamientos entre estudiantes que están viviendo una realidad y que son conscientes y sensibles a todo lo que ocurre en ella.

"El aislamiento del profesor en su aula trabaja en contra de la reflexión desde la acción. Necesita comunicar sus problemas y percepciones, para contrastarlos con los puntos de vistas de sus colegas" (Schön, 1998, p. 290). El autor rescata el hecho de que socializar la cotidianidad de las labores como docentes entre colegas, puede generar situaciones de aprendizaje más ricas y variadas, pues enfrentamos nuestras dudas y miedos, ponemos a prueba en frente de otros que se enfrentan a situaciones de aula similares, nuestros esquemas de acción, nuestras posturas teóricas que emergen en la práctica y que 
constituyen de alguna manera nuestra identidad como maestros; identidad que en la mayoría de los casos evitamos exponer ante otros para no ser evaluados, y con esa actitud se aleja la oportunidad de reconstruir las acciones en conjunto dentro de una comunidad de colegas, y el fomentar la cultura de la socialización de las experiencias libres del sentimiento de ser evaluados que debe estar presente en las aulas universitarias.

Dentro de las reflexiones la idea de acercarse a la formación de un profesor investigador consciente de sus acciones y de la relación teoría-práctica, a partir de los espacios iniciales de la intervención pedagógica, es otro de los retos importantes. Como apunta Bondarenko (2009), los profesores formadores deberían de considerar si desean estudiantes consumidores o repetidores de teorías y conocimientos desde un discurso predeterminado, o si quieren estudiantes, que construyan sus propias teorías, a partir de la reflexión de sus prácticas.

La investigación fortalece y empodera al docente en formación en su propio proceso, pues es el que reflexiona, replantea y toma decisiones. La figura de un profesor investigador permitiría pensar en un estudiante que construya sus propios caminos hacia el conocimiento, eliminado la dependencia cognitiva que se manifiesta a lo largo de los diferentes procesos en los cuales se le acompaña, pues estaría asumiendo la responsabilidad de tomar decisiones durante y después de sus acciones producto de un empoderamiento que solo emerge cuando hay certeza de conocimiento de la realidad en la que se desenvuelve, producto de la revisión y el análisis constante de su acción.

La respuesta a estos retos deberá surgir, nuevamente, de un proceso de reflexión que lleve a trazar rutas de acción que serán sujeto de nuevas reflexiones, para generar prácticas más cercanas a formar docentes con habilidades críticas profundas, y de prácticas con sentido producto de sus propias reflexiones. Una vez más, emerge la necesidad de voltear la mirada más allá de lo cotidiano de nuestras prácticas como profesores formadores de futuros docentes.

\section{REFERENCIAS}

Bondarenko, N. (2009). El componente investigativo y la formación docente en Venezuela. Revista Estudios Pedagógicos XXXV, N. 1: 253- 260. Recuperado de http://mingaonline.uach.cl/scielo.phap

Gimeno, J. y Pérez, A. (2000). Comprender y transformar la enseñanza. Madrid, España.

Jackson, W. (1998). La vida en las aulas. Madrid, España: Ediciones Morata.

Schön, D. (1998). El profesional reflexivo: Cómo piensan los profesionales cuando actúan. Barcelona, España: Editorial Paidós.

Sanjurjo, L. (2002). La formación práctica de los docentes, la reflexión y acción en el aula. Rosario, Argentina: Homo Sapiens Ediciones.

Sanjurjo, L., Hernández, A., España, E., Caporossi, A., Alonso, I., y Foresi, M. (2009). Los dispositivos para la formación de las prácticas profesionales. Rosario, Argentina: Homo Sapiens Ediciones.

Sierra, J., Escudero, J; Sabirón, F; Pérez, J; Martínez, J; Alcalá, M; Rodríguez, S; Padilla, E; Montecillo, M. (2002). Evaluación del rendimiento, evaluación del aprendizaje. Universidad Internacional de Andalucía. Ediciones Akal. Madrid, Epaña.

Universidad Nacional (UNA). (2012). Áreas y Principios de la carrera de Licenciatura en pedagogía con énfasis en Educación Preescolar. Recuperado de htt://www. cide-basica.una.ac.cr/index.php?opcion $=$ com_remo sitory\&Itemid $=77 \&$ func $=$ startdown $\&$ id $=48$

Universidad Nacional (UNA). (2012).Descriptores de cursos carrera preescolar. Recuperado de http:// www.cide-basica.una.ac.cr/index.php?option $=\mathrm{com}_{-}$ remository\&Itemid $=77 \&$ func $=$ startdown $\&$ id $=40$ 
